\title{
Ungenauigkeit der Interozeption und Abwendung der Aufmerksamkeit bei Atemwegserkrankungen: Asthma bronchiale versus chronisch obstruktive Bronchitis
}

\author{
Accuracy of Interoception and Withdrawal of Attention in Airway Diseases: Bronchial \\ Asthma versus Chronic Obstructive Bronchitis
}

\author{
J. Hoyera A. Reusch ${ }^{b} \quad$ E. Leibing ${ }^{c}$ \\ aTU Dresden, Klinische Psychologie und Psychotherapie, ${ }^{b}$ Rehabilitationswissenschaftlicher Forschungsverbund Bayern, Würzburg, \\ 'A Abteilung für Psychotherapie und Psychosomatik, Georg-August-Universität Göttingen
}

\section{Schlüsselwörter \\ Aufmerksamkeit · Interozeption · Atemwegserkrankungen · Asthma bronchiale . Chronisch obstruktive Bronchitis}

\section{Zusammenfassung}

In der vorliegenden Studie wurde die Hypothese geprüft, daß Asthmatiker die Aufmerksamkeit von eigenen Körperprozessen ablenken und eine Ungenauigkeit bei der Interozeption relevanter Atemwegsobstruktionen aufweisen. Weiterhin prüften wir die Frage, inwieweit die postulierte Aufmerksamkeitsabwendung generalisiert ist und sich auch auf die nicht atemwegsbezogene Symptomwahrnehmung und die private Selbstaufmerksamkeit bezieht. Die Interozeptionsgenauigkeit wurde als Diskrepanz zwischen subjektivem Urteil und objektiver Atemfunktion bei spirometrischen Messungen berechnet, die anderen Variablen mittels Fragebögen operationalisiert. Es wurden insgesamt 91 Patienten einer Rehabilitationseinrichtung untersucht: 30 Asthmatiker, 30 Patienten mit chronisch obstruktiver Bronchitis (COB) und 31 Kontrollpatienten ohne Atemwegserkrankung. Die Ergebnisse deuten auf eine spezifisch atemwegsbezogene Aufmerksamkeitsablenkung sowie eine Überschätzung von Obstruktionen bei Asthmatikern hin. Überraschend zeigen auch die COB-Patienten auffällige Ergebnismuster in Richtung einer Unterschätzung von Obstruktionen sowie verminderter Selbstaufmerksamkeit. Die Ergebnisse lassen sich im Rahmen verhaltensmedizinischer Überlegungen interpretieren.

\section{Key Words}

Attention - Interoception - Airway diseases ·

Bronchial asthma · Chronic obstructive bronchitis

\section{Summary}

The hypothesis that asthmatic patients draw their attention away from bodily processes and show inaccurate interoception with regard to relevant airway obstructions was tested in this study. Additionally, we examined whether this postulated withdrawal of attention can also be generalized for the perception of non-airway related symptoms as well as for private self-consciousness. Accuracy of interoception was measured as the discrepancy between subjective judgement of obstruction and objective obstruction as shown in spirometric tests. Other variables were operationalized by self-reports. Ninetyone patients in a rehabilitation hospital were tested: 30 asthmatic patients, 30 patients with chronic obstructive bronchitis (COB), and 31 control subjects without any airway disease. Asthmatic patients showed attention withdrawal only with regard to bronchial airways. However, they also indicated an overestimation of airway obstruction. Surprisingly, deviant results were also found for the $\mathrm{COB}$ patients including underestimation of obstructions and lower self awareness. All results were interpreted from the perspective of behavioral medicine.

\begin{tabular}{ll}
\hline KARGER & ๑ 1999 S. K arger G mbH , F reiburg \\
Fax +49 761 452 07 14 & A ccessible online at: \\
$\begin{array}{l}\text { E-mail kargergmbh@aol.com } \\
\text { www.karger.com }\end{array}$ & http://B ioM edN et.com/karger
\end{tabular}

\section{KARGER}

E-mail kargergmbh@aol.com www.karger.com

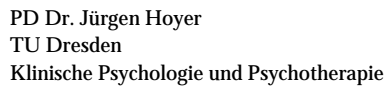




\section{Einleitung}

Bei chronischen Erkrankungen ist die Wahrnehmung von Veränderungen im E rkrankungsgeschehen von zentraler B edeutung für das Verhaltensmanagement. Gegensteuernde $M$ aßnahmen setzen voraus, daß symptomatische Veränderungen rechtzeitig und genau erkannt werden. Im Fall asthmatischer Erkrankungen besteht deshalb ein Behandlungsziel in der E rhöhung der Sensibilität für leichte respiratorische E inschränkungen durch das Training der A tmungswahrnehmung [z.B. Könning et al., 1996; Stout et al., 1993; 1997]. Der Ausbildung einer vollständigen A sthma-A ttacke soll so rechtzeitig entgegengewirkt werden. A llerdings sind nicht nur U nterschätzungen atemwegsbezogener Obstruktionen, sondern auch deren Ü berschätzungen ungünstig: Ü berschätzen Patienten einen A sthmaanfall und stufen ihre B ewältigungsmöglichkeiten als gering ein, so intensiviert sich die emotional negative $\mathrm{R}$ eaktion, was wiederum die $\mathrm{O}$ bstruktion der $\mathrm{A}$ temwege verstärken kann [vgl. Kosarz, 1994].

In diesem Zusammenhang kommt der Frage Bedeutung zu, ob A sthmatiker den Schweregrad einer aktuellen O bstruktion realistisch einschätzen können. Mehrere experimentelle B efunde sprechen für eine geringere Interozeptionsgenauigkeit von A sthmatikern. Dahme et al. [1991] fanden in drei unabhängigen Studien bei A sthmatikern zunächst, daß diese in der Interozeption der durch einen Allergentest provozierten $\mathrm{O}$ bstruktionen ungenau und unsicher waren. Sie neigten in der Mehrzahl zur U nterschätzung (D issimulation) der Obstruktion. In dieser Studie fehlte jedoch eine Kontrollgruppe. Zwei weitere experimentelle Studien, in denen ein externer A temwegswiderstand (Siebwiderstand) zur O bstruktionserzeugung eingesetzt wurde, erbrachten widersprüchliche Ergebnisse. Welche Gruppe (unabhängig von A ntworttendenzen) bessere Sensitivitätskennwerte im Sinne korrekter Interozeption erreichte, hing letztlich von der Auswertungsmethode $a b$. E in weiteres Problem der letztgenannten Studien ist ihre fragliche ökologische Validität: A sthmatiker haben es eben nicht mit externen A temwegsobstruktionen zu tun. Prozesse, die bei der Interozeptionsverzerrung eine R olle spielen könnten - z.B. die A ngst vor einem bevorstehenden A nfall - bleiben bei solchen nicht alltagsrelevanten Vorgehensweisen ausgeklammert. Die A utoren selbst fordern deshalb weitere $U$ ntersuchungen.

In der vorliegenden Studie soll die Wahrnehmung von A temwegsobstruktionen bei A sthmatikern mit einer veränderten M ethodik untersucht werden. D ie Versuchsteilnehmer sollen ihre Schätzurteile nicht auf externale, «künstliche» A temwegswiderstände beziehen, sondern auf ihre aktuellen, durch das $\mathrm{K}$ rankheitsgeschehen bedingten «internalen» 0 bstruktionen. U m die G enauigkeit der Schätzurteile zu erfassen, wird die subjektive Einschätzung der aktuellen Obstruktion mit der am Normwert geeichten A temstromstärke bzw. Sekundenkapazität in Beziehung gesetzt (E rläuterungen s. unten). A nalog zu bisherigen, methodisch divergenten Stu- dien, erwarten wir dabei in $\mathrm{H}$ ypothese 1 , daß A sthmatiker gegenüber einer Kontrollgruppe eine verminderte Interozeptionsgenauigkeit bezüglich der A temwegsobstruktion aufweisen.

Weiterhin soll in der Studie die Frage geprüft werden, inwieweit A sthmatiker nicht nur eine verringerte Interozeptionsgenauigkeit bezüglich der A temwege, sondern auch eine generalisierte Vermeidung von Symptom- oder Selbst-Wahrnehmungen aufweisen. Werden körperliche Symptome nicht nur beim erkrankten, sondern auch bei anderen Organen ungenauer wahrgenommen ( $H$ ypothese 2 )? Sachse [1994] fand unter dieser $\mathrm{H}$ ypothese bei einer diagnostisch heterogenen Stichprobe von «psychosomatischen Patienten», daß die multimodal gemessene $\mathrm{H}$ erzschlagwahrnehmung ungenauer als bei einer Kontrollgruppe war.

Ferner ist zu fragen (H ypothese 3), ob - über die defizitäre Symptomwahrnehmung hinaus - die Selbstwahrnehmung allgemein weniger deutlich ist, ob also die D isposition zur «privaten Selbstaufmerksamkeit» [Fenigstein et al., 1975; Filipp und Freudenberg, 1989; Filipp et al., 1993] herabgesetzt ist, so wie das von Sachse [1995] für die sogenannten «psychosomatischen Störungen» vermutet wird [vgl. Befunde von Sachse und R udolph, 1992; Sachse, 1994]. D anach wäre gleichzeitig die «öffentliche Selbstaufmerksamkeit», also die Tendenz, darauf zu fokussieren, was andere von einem denken, gesteigert. Die Grundlage dieser theoretischen Ü berlegung ist, daß anders als im A lexithymie-K onzept [G erhards, 1988], psychosomatisch gestörte Patienten zwar kein grundsätzliches D efizit in der Wahrnehmung von internalen Reaktionen und E motionen aufweisen, diese Wahrnehmungen aber unter den Bedingungen einer Wahrnehmungsabwehr stehen. Eigene Bedürfnisse, körperliche wie psychische, werden deswegen weniger wahrgenommen und das Verhalten vermehrt aufgrund sozialer Standards gesteuert.

Die U ntersuchung der genannten Fragestellungen soll in einem klinischen Kontrollgruppendesign vorgenommen werden: A sthmapatienten sollen nicht nur mit atemwegsgesunden $\mathrm{K}$ linikpatienten, sondern auch mit einer klinischen, ebenfalls atemwegserkrankten Kontrollgruppe verglichen werden. D ieser Vergleich erlaubt die Beurteilung, ob die postulierten A bweichungen zu den spezifischen M erkmalen von A sthmaerkrankungen gehören oder ob sie allgemein als Korrelat von A temwegserkrankungen einzustufen sind.

A Is klinische Vergleichsgruppe bietet sich das Störungsbild der chronisch obstruktiven B ronchitis (COB) an. Die COB ist eine $\mathrm{A}$ temwegserkrankung, die in $80 \%$ der Fälle durch $\mathrm{Ni-}$ kotinkonsum verursacht wird und bei der eine chronische Entzündung und Schwellung der Bronchialschleimhaut besteht, die ähnlich dem A sthma mit einer A temwegsobstruktion und subjektiver $A$ temnot einhergeht. I $\mathrm{m} U$ nterschied zum A sthma, bei dem die $O$ bstruktion anfallsweise und reversibel verläuft, handelt es sich bei der COB um eine chronisch progrediente $\mathrm{O}$ bstruktion, die nur bedingt medikamentös behandelt werden kann. 
Tab. 1. Soziodemographische und erkrankungsbezogene $D$ aten der U ntersuchungsgruppen ( $H$ äufigkeiten bzw. Mittelwerte und Standardabweichungen) und E rgebnisse der Tests auf Stichprobenhomogenität

\begin{tabular}{|c|c|c|c|c|c|}
\hline & $\begin{array}{l}\text { A sthma-Patienten } \\
(n=30)\end{array}$ & $\begin{array}{l}\text { COB-Patienten } \\
(n=30)\end{array}$ & $\begin{array}{l}\text { Kontrollgruppe }{ }^{b} \\
(n=31)\end{array}$ & $\chi^{2} / F / t^{a}$ & $\mathrm{p}$ \\
\hline G eschlecht (m/w) & $18 / 12$ & $20 / 10$ & $20 / 11$ & 0,30 & 0,86 \\
\hline A lter, M W (SD ), Jahre & $46,9(13,2)$ & $50,5(8,48)$ & $51,9(8,9)$ & 1,85 & 0,17 \\
\hline \multicolumn{4}{|l|}{ Partnerschaft, n (\%) } & \multirow[t]{3}{*}{1,95} & \multirow[t]{3}{*}{0,38} \\
\hline mit Partner & $22(73,3)$ & $26(86,7)$ & $23(74,2)$ & & \\
\hline ohne Partner & $8(24,7)$ & $4(13,4)$ & $8(25,8)$ & & \\
\hline \multicolumn{4}{|l|}{ Schulbildung, n (\%) } & \multirow[t]{4}{*}{2,31} & \multirow[t]{4}{*}{0,31} \\
\hline H auptschule & $26(86,7)$ & $28(93,3)$ & $23(74,2)$ & & \\
\hline R ealschule & $4(13,3)$ & $2(6,7)$ & $6(19,4)$ & & \\
\hline A bitur & 0 & 0 & $2(6,5)$ & & \\
\hline Erkrankungsdauer, Jahre & $14,6(11,9)$ & $10,1(12,3)$ & & 2,11 & 0,15 \\
\hline Erkrankungsalter & $32,4(20,1)$ & $40,3(13,9)$ & & 3,13 & 0,08 \\
\hline \multicolumn{4}{|l|}{ Schweregrad, n (\%) } & \multirow[t]{3}{*}{0,30} & \multirow[t]{3}{*}{0,86} \\
\hline mittel & $20(66,7)$ & $22(73,3)$ & & & \\
\hline schwer & $10(33,3)$ & $8(26,7)$ & & & \\
\hline \multicolumn{3}{|c|}{ Subjektive B eeinträchtigung, $n(\%)$} & & \multirow[t]{4}{*}{3,12} & \multirow[t]{4}{*}{0,21} \\
\hline ein wenig, & $4(13,3)$ & $8(26,6)$ & & & \\
\hline ziemlich & $14(46,7)$ & $8(26,7)$ & & & \\
\hline sehr & $12(40)$ & $14(46,7)$ & & & \\
\hline
\end{tabular}

D a für die Prüfung der ersten $\mathrm{H}$ ypothese die atemwegsgesunde Kontrollgruppe nicht zur Verfügung stand (s. u.), lauten die zu prüfenden $\mathrm{H}$ ypothesen wie folgt:

1. A sthmatiker weisen eine geringere Interozeptionsgenauigkeit als CO B-Patienten auf.

2. Sie nehmen subjektiv ihre Symptome weniger genau wahr als CO B -Patienten und atemwegsgesunde Kontrollpatienten.

3. Sie zeigen geringere private und höhere öffentliche Selbstaufmerksamkeit als beide Kontrollgruppen.

\section{Methoden}

Stichproben

A Is Probanden wurden Patienten der R ehabilitationsklinik «A m Taunus» in Bad Soden, einer internistischen K linik mit dem Schwerpunkt Pneumologie, rekrutiert. Diese K linik wird größtenteils durch Patienten der $L$ andesversicherungsanstalten von Baden-Würtemberg und $B$ ayern belegt. Die untersuchten Stichproben sind somit im $\mathrm{H}$ inblick auf den sozioökonomischen Status (vorwiegend A rbeiter), das A Iter (relativ hoch) und die soziokulturelle $R$ egion homogen, aber nur eingeschränkt repräsentativ. E s wurden insgesamt $n=91$ Patienten untersucht; davon je 30 in der A sthma- und in der COB-G ruppe und 31 in der atemwegsgesunden Kontrollgruppe. Die Zuweisung zu den Diagnosegruppen wurde gemäß der ärztlich-diagnostischen U ntersuchung vorgenommen. A Is A usschlußkriterium galt jeweils, daß keine eindeutige Zuordnung zu den $G$ ruppen vorgenommen werden konnte ( $C O B$ - und A sthmadiagnose überschneiden sich häufig) und daß D eutsch nicht M uttersprache war. Probanden der Kontrollgruppe ( $K G$ ) sollten keine A temwegserkrankung aufweisen. $D$ ie $G$ ruppe der A sthmatiker setzte sich aus den Diagnosegruppen extrinsisches $(n=9)$, intrinisches $(n=11)$ und gemischtförmiges $(n=10)$
A sthma zusammen. Die Patienten in der K G wiesen zu 93,2\% degenerative $E$ rkrankungen im B ereich der Wirbelsäulen und $\mathrm{G}$ elenke auf; ein $\mathrm{Pa}$ tient litt an Schlafapnoe, einer an Sinusitis.

A ngaben zu wesentlichen soziodemographischen und erkrankungsbezogenen $D$ aten der Stichproben sind Tabelle 1 zu entnehmen.

$D$ as $G$ eschlechterverhältnis liegt in allen drei Gruppen bei etwa 2:1 (M änner zu Frauen), was dem üblichen B elegungsverhältnis der K linik entspricht. $D$ as durchschnittliche $A$ Iter der Stichproben liegt entweder knapp unter (A sthma), oder leicht über einem Wert von 50 Jahren. In allen drei Stichproben leben jeweils etwa 3/4 der Probanden in festen Partnerschaften; die meisten Probanden weisen einen $\mathrm{H}$ auptschulabschluß auf. $\mathrm{H}$ insichtlich dieser Variablen ergeben sich keine statistisch bedeutsamen Stichprobenunterschiede.

Bezogen auf das E rkrankungsalter und die Erkrankungsdauer gilt, daß A sthmatiker verglichen mit $\mathrm{CO} B$-Patienten tendenziell früher und länger erkrankt sind (vgl. Tab. 1, p jeweils $<0,15$ ), was für die Störungsbilder repräsentativ ist. In Vorprüfungen ergaben sich jedoch keine bedeutsamen Zusammenhänge dieser beiden potentiellen Störvariablen mit den abhängigen Variablen der U ntersuchung.

Weiterhin wurde der Schweregrad der E rkrankung gemäß ärztlicher D iagnose ( «Schweregrad») und subjektiver E inschätzung der Beeinträchtigung erfaßt. Subjektiv erleben sich 40 (A sthma) bzw. 46,7\% (COB) der Probanden als sehr beeinträchtigt; nach ärztlicher Diagnose gemäß Ferlinz [1992] wird jeweils knapp ein D rittel der Probanden als schwer erkrankt eingestuft. Relevante Gruppenunterschiede ergeben sich nicht. Spezifischere krankheitsbezogene $D$ aten zu den Stichproben sind in R eusch [1997] dargestellt.

\section{M eßinstrumente}

E rfassung der atemwegsbezogenen Interozeptionsgenauigkeit. In der experimentellen Forschung zur $\mathrm{O}$ bstruktionswahrnehmung werden häufig extern in den A temstrom eingesetzte Siebwiderstände verwendet und deren objektiv bekannte $\mathrm{G}$ röße wird zu der von den Probanden subjektiv wahrgenommenen 0 bstruktion ins Verhältnis gesetzt [vgl. z.B. D ahme et 
al., 1991]. Ein Nachteil dieser Methodik liegt in der fraglichen ökologischen Validität. Es ist unklar, ob sich die Ergebnisse auf symptomatische Bronchokonstriktionen übertragen lassen. In der vorliegenden A rbeit wurde deshalb die subjektive Einschätzung des aktuellen symptomatischen $\mathrm{Obstruktionsgrades} \mathrm{erfaßt} \mathrm{und} \mathrm{mit} \mathrm{einem} \mathrm{direkt} \mathrm{im}$ A nschluß gemessenen objektiven Parameter verglichen.

Hierzu wurde das sogenannte Tiffeneau-A temmanöver der Lungenfunktionsdiagnostik durchgeführt, das eine Orientierung über den Grad der Strömungsbehinderung im Bronchialsystem ermöglicht: Die Patienten werden aufgefordert, am Spirometer (G erät zur M essung der Stärke des A temflusses) zunächst langsam maximal aus-, dann einzuatmen, dann den A tem kurz anzuhalten und schließlich so schnell wie möglich wieder auszuatmen. D er gemessene 0 bstruktionsgrad ist um so höher, je mehr die maximale A temstromstärke (peak expiratory flow, PE F) eingeschränkt ist oder je weniger A temluft innerhalb der ersten Sekunde ausgeatmet werden kann (Sekundenkapazität, forced expiratory volume, FEV). Ü blicherweise werden die ermittelten PEV- und FEV-Werte in Prozent der Sollwerte einer gesunden Normwertegruppe ausgedrückt $\left(P E V_{\text {Soll\%, }}, F V_{\text {Soll\% }}\right)$. Z ur E rfassung der PE V- und FE V-Werte sowie der entsprechenden Sollprozentwerte wurde ein Pneumoscope ${ }^{\circledR}$ (Version 2.1) der Firma E rich J äger ( $H$ öchberg, D eutschland) eingesetzt. $D$ ie vom G erät gelieferten N ormwerte basieren auf den D aten von Q uanjer [1983] und orientieren sich an den Parametern A Iter, G eschlecht, Körpergröße und Körpergewicht.

D ie subjektive $E$ inschätzung der aktuellen $A$ temwegsobstruktion erfolgte mittels einer visuellen A nalogskala. U m Carry-over-E ffekte zu vermeiden, konnte hier nicht die oft verwendete Kodierung von $0-100 \%$ gewählt werden, da den Patienten üblicherweise in der Eingangsdiagnostik die mit dem Spirometer erfaßten Sollprozentwerte mitgeteilt werden. In A nlehnung an eine Skala von Löllgen und Dirschedl (R atings of Perceived Dyspnoe, R PD) [1992] erhielten die Patienten statt dessen die Instruktion, ihre aktuelle $A$ nstrengung beim $A$ tmen einzuschätzen. E s wurde eine intermittierend verbal kodierte Skala mit 15 Teilstrichen von 6 («keinerlei A nstrengung») bis 20 («zu stark, geht nicht mehr») vorgegeben. A nschließend wurden die Werte invertiert und in Prozentwerte (RPD - $1 \%$ ) umgerechnet, da es sich bei den spirometrischen Werten um Funktionswerte handelt und die Schätzurteile mit diesen vergleichbar werden sollten

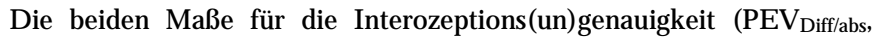
$F E V_{D \text { iff/abs }}$ ) errechnen sich aus der absoluten Differenz zwischen dem mit dem Pneumoscope ermittelten objektiven ( $P E V_{\text {soll\%, }}, \mathrm{FEV}_{\text {Soll\% }}$ ) und dem subjektiv eingeschätzten, in Prozentwerte transformierten Wert (RPD $-1 \%$ ) der A temwegsfunktion (Formel 1 ):

a) $P E V_{\text {Diff/abs }}=\left|P E V_{\text {Soll }} \%-R P D-\right| \% \mid$

b) $F E V_{\text {Diff/abs }}=\left|F E V_{\text {soll } \%}-R P D-\right| \% \mid$.

Je größer $P E V_{D \text { iff/abs }}$ und $F E V_{D}$ iff/abs, d.h. die $D$ ifferenz zwischen dem objektiven $\mathrm{M}$ aß und der subjektiven $\mathrm{E}$ inschätzung der $\mathrm{A}$ temwegsfunktion ist, desto ungenauer ist die Interozeptionsleistung.

Wahrnehmung körperlicher Symptome.

Im «Fragebogen zur Wahrnehmung körperlicher Symptome» (FWKS) von E rdmann und Janke [1975] werden A ussagesätze zur K örpersel bstbeobachtung und der K örperwahrnehmung in einem dichotomen A ntwortmodus dargeboten. In der vorliegenden A rbeit werden aus ökonomischen $G$ ründen nur drei der insgesamt acht Skalen des Fragebogens eingesetzt: D ie Skala zur A tmung (FWK S-A, 10 Items, B eispiel: «A bends vor dem E inschlafen höre ich mir häufiger beim A tmen zu»), die Skala zur Wahrnehmung allgemeiner körperlicher Symptome (FWK S-KS, 15 Items, B eispiel: «I ch merke sofort, wenn mit meinem Körper etwas nicht stimmt») und, um auch die Wahrnehmung an einem spezifischen, in unseren Stichproben erkrankungsfremden O rgan zu erfassen, die Skala für den gastro-intestinalen Trakt (FWK S-GIT, 10 Items, B eispiel: «In aufregenden Situationen achte ich manchmal auf Veränderungen in der $\mathrm{Ma-}$ gengegend»). H euser [1991] berichtet für die genannten Skalen mäßige bis gute Werte für die interne Konsistenz an einer Stichprobe von A Iko- holikern (FWK S-A : $\alpha=0,70$; FW K S-K S: $\alpha=0,76$; FWK S-G IT: $\alpha=0,82$ ). In den Items werden keineswegs ausschließlich Fragen nach «Symptomen» formuliert, denn es überwiegen Items, die auf die $\mathrm{N}$ eigung abzielen, non-symptomatische körperbezogene Empfindungen und Wahrnehmungen zu fokussieren (z.B.: «lch beobachte selten, ob ich körperlich irgendwie reagiere»; ein invers gepoltes Item). Der Fragebogen erfaßt, ganz im Sinne der Intention der eigenen Studie, also eher eine Tendenz zu körperbezogener Vigilanz. Für den Fragebogen liegen zwar weder Normwerte noch befriedigende $D$ aten zur Konstruktvalidität vor, er ist jedoch augenscheinvalide und gut handhabbar; zudem liegen unseres Wissens keine besser geeigneten $\mathrm{M}$ eßmethoden vor.

\section{Selbstaufmerksamkeit}

$M$ it dem «Fragebogen zur E rfassung der D ispositionellen Selbstaufmerksamkeit» (SA M -Fragebogen) von Filipp und Freudenberg [1989] wurden die private (13 Items) und öffentliche (14 Items) Selbstaufmerksamkeit gemessen. $\mathrm{Zu}$ diesem Instrument liegen im Gegensatz zu anderen deutschsprachigen B earbeitungen der Self-Consciousness Scale [SCS, Fenigstein et al., 1975; z.B. M erz, 1986] nicht nur befriedigende testtheoretische K ennwerte, sondern auch $N$ ormen vor.

\section{D urchführung/U ntersuchungsablauf}

$\mathrm{Nach}$ der medizinisch-diagnostischen Untersuchung in der ersten Behandlungswoche wurden in der zweiten Woche die E inwilligung der Patienten eingeholt und die soziodemographischen und krankheitsbezogenen $D$ aten erfaßt. Die M essungen zur Interozeptionsgüte sowie die Fragebogenerhebungen wurden jeweils in der dritten Behandlungswoche in K leingruppen durchgeführt. Tageszeit und $\mathrm{Ort}$ wurden für alle Datenerhebungen konstant gehalten. Es gab keine $\mathrm{H}$ inweise auf M otivationsunterschiede bei der D urchführung des Tiffeneau-M anövers.

\section{Statistische A nalysemethoden}

Für die Prüfung der Hypothesen über Gruppenunterschiede wurden tTests eingesetzt, da entsprechend spezifizierte $H$ ypothesen über einzelne $\mathrm{G}$ ruppenvergleiche vorliegen.

\section{Ergebnisse}

Deskriptive und hypothesenprüfende Ergebnisse zu den U nterschiedshypothesen werden gemeinsam dargestellt; sie sind in Tabelle 2 einzusehen.

\section{Interozeptionsgüte.}

D ie Interozeptionsgüte wurde pro Patient über die absoluten Differenzwerte zwischen subjektiver E inschätzung und objektiv meßbarer A temfunktion operationalisiert (vgl. Formel 1). U nabhängig davon, ob A temstromstärke oder Sekundenkapazität betrachtet werden, liegen die Indikatoren der Interozeptionsgüte bei A sthma- und COB-Patienten offensichtlich in gleicher $\mathrm{H}$ öhe (Tab. 2).

Der eingeschätzte Wert weicht in beiden $M$ aßen und bei beiden $\mathrm{G}$ ruppen durchschnittlich etwa $15 \%$ vom objektiv gemessenen Funktionswert $a b$. Demgemäß ergeben sich keine $U$ nterschiede im Signifikanztest. Die erste $\mathrm{H}$ ypothese ist damit zurückzuweisen. A sthmatiker zeigen gegenüber einer Gruppe von Patienten mit einer anderen A temwegserkrankung keine ungenaueren I nterozeptionswerte.

U $\mathrm{m}$ A ussagen über die Richtung der $\mathrm{A}$ bweichungen machen 
Tab. 2. M ittelwerte und Standardabweichungen in den U ntersuchungsvariablen und E rgebnisse der geplanten und explorativen (kursiv) statistischen

G ruppenvergleiche zwischen

A sthmatikern (I), COB-Patienten (II) und Kontrollprobanden (III)

\begin{tabular}{|c|c|c|c|c|c|c|}
\hline Variable & $\begin{array}{l}\text { A sthma-Patienten } \\
(n=30)\end{array}$ & $\begin{array}{l}\text { COB-Patienten } \\
(n=30)\end{array}$ & $\begin{array}{l}\text { Kontrollgruppe } \\
(n=31)\end{array}$ & Vergleich & $\mathrm{t}$ & $p$ \\
\hline |PE V-D iff| & $15,73(10,39)$ & $13,30(10,85)$ & - & $1 / I I$ & $-0,89$ & 0,38 \\
\hline |FEV-D iff| & $16,19(10,41)$ & $16,47(9,83)$ & - & $1 / I I$ & 0,11 & 0,91 \\
\hline PE V-D iff & $8,60(16,96)$ & $-0,81(17,32)$ & - & $I / I I$ & 2,20 & $0,04^{a}$ \\
\hline FE V-D iff & $6,03(18,49)$ & $-6,16(18,39)$ & - & $1 / I I$ & 2,50 & $0,01^{a}$ \\
\hline \multirow[t]{3}{*}{ FWK S-A } & $1,42(0,21)$ & $1,52(0,24)$ & $1,63(0,26)$ & $1 / I I$ & $-1,61$ & 0,11 \\
\hline & & & & $\mid / I I I$ & $-3,30$ & 0,00 \\
\hline & & & & $|I / I I|$ & $-1,59$ & 0,12 \\
\hline \multirow{3}{*}{ FWK S-KS } & $1,39(0,25)$ & $1,54(0,24)$ & $1,52(0,26)$ & $1 / I I$ & $-2,23$ & 0,03 \\
\hline & & & & $|/ I I|$ & $-2,02$ & 0,01 \\
\hline & & & & $\mid I / I I I$ & 0,23 & 0,82 \\
\hline \multirow[t]{3}{*}{ FWK S-GIT } & $1,45(0,23)$ & $1,54(0,20)$ & $1,51(0,27)$ & $1 / I I$ & 1,66 & 0,10 \\
\hline & & & & $|/ I I|$ & $-1,02$ & 0,31 \\
\hline & & & & $|I / I I|$ & 0,44 & 0,66 \\
\hline \multirow[t]{3}{*}{ SA M -P } & $46,70(7,65)$ & $41,23(6,64)$ & $47,29(9,40)$ & $1 / I I$ & 2,70 & 0,01 \\
\hline & & & & $\mid / I I I$ & -.24 & 0,81 \\
\hline & & & & $\|/\| I \mid$ & 2,90 & 0,00 \\
\hline \multirow[t]{3}{*}{ SA M -Ö } & $46,63(7,33)$ & $41,70(8,53)$ & $44,29(8,72)$ & $1 / I I$ & 2,33 & 0,00 \\
\hline & & & & $|/ I I|$ & 1,11 & 0,27 \\
\hline & & & & $\|/\| \|$ & $-1,17$ & 0,25 \\
\hline
\end{tabular}

FWKS = Fragebogen zur Wahrnehmung körperbezogener Symptome; FWKS-A = Skala atmungsbezogene Symptome; FWK S-KS = Skala allgemeine Symptome; FWK S-GIT = Skala gastrointestinale Symptome; PEV-D iff = A bweichung der subjektiven und der am Normwert geeichten (objektiven) A temstromstärke; FE V-D iff = A bweichung der subjektiven und der am N ormwert geeichten (objektiven) Sekundenkapazität; für PE V-D iff und FEV-D iff werden jeweils der absolute und der relative $\mathrm{A}$ bweichungswert angegeben. aZ weiseitiger Test. zu können, wurden auch $D$ ifferenzwerte berechnet, bei denen das Vorzeichen berücksichtigt wurde. E in Differenzwert mit positivem Vorzeichen (objektiver Sollprozentwert > subjektive Einschätzung) bedeutet damit ein Unterschätzen der A temwegsfunktion bzw. eine Ü berschätzung der obstruktiven Symptomatik. Es fällt auf, daß das Vorzeichen bei A sthmatikern jeweils positiv ist; sie weisen also eine relative Ü berschätzung der O bstruktion auf. B ei COB-Patienten hingegen sind die Vorzeichen nahe null bzw. negativ, was eine relative Unterschätzung der $\mathrm{O}$ bstruktion bedeutet. $\mathrm{D}$ ie a posterioriUnterschiedsprüfung zeigt, daß diese Unterschiede signifikant sind. $U \mathrm{~m}$ auszuschließen, daß diese E rgebnisse auf einzelne «A usreißer» zurückgehen, wurden zusätzlich die Probanden danach eingeteilt, ob sie eine positive oder negative A bweichung ihrer $E$ inschätzung aufweisen und anschließend ein $\chi^{2}$-Test durchgeführt. Bei $P E V_{D i f f}$ finden sich in der A sthmatiker-G ruppe $n=23$, bei $F E V_{\text {Diff }} n=22$ Ü berschätzer (der 0 bstruktion) und $n=7$ bzw. $n=8 U$ nterschätzer. B ei den COB-Patienten gibt es bei PEV $V_{\text {Diff }} 17$ Über- und 13 Unterschätzer, bei $F E V_{\text {Diff }} 10$ Ü berschätzer und 20 U nterschätzer. $B$ ei dieser nominalskalierten Betrachtung wird der Gruppenunterschied für $F E V_{\text {Diff }}$ signifikant $\left(\chi^{2}=9,64 ; p=0,002\right)$, für $P E V_{D i f f}$ findet sich eine statistische Tendenz $\left(\chi^{2}=2,70 ; p=\right.$ $0,10)$.

Wahrnehmung körperbezogener Symptome

Die Gruppenmittelwerte im Fragebogen zur Wahrnehmung körperlicher Symptome (FWKS) sind bei A sthmatikern je- weils am niedrigsten, sowohl bei den atmungsbezogenen Symptomen (FWK S-A) als auch bei den allgemeinen Symptomen (FWK S-KS). B ei der FWK S-A zeigen die einseitigen t-Tests für den Vergleich mit der $\mathrm{COB}-\mathrm{G}$ ruppe jedoch nur einen nichtsignifikanten U nterschied an; der Vergleich zur Kontrollgruppe wird signifikant. Die zweite $\mathrm{H}$ ypothese hat sich damit teilweise bestätigt; A sthmatiker beobachten atmungsbezogene $\mathrm{K}$ örpersymptome weniger intensiv als $\mathrm{A}$ tmungsgesunde.

Auch die Wahrnehmung sonstiger körperbezogener Symptome (FW K S-K S) ist bei den A sthmatikern signifikant geringer als in den beiden anderen $G$ ruppen. B etrachtet man mit der Symptomwahrnehmung für den Gastrointestinaltrakt (FWKS-GIT) einen spezifischen krankheitsfremden Bereich, so finden sich keine signifikanten $U$ nterschiede zwischen den Gruppen.

\section{Selbstaufmerksamkeit}

D ie G ruppenmittelwerte in privater und öffentlicher Selbstaufmerksamkeit sind sowohl in der A sthmatiker-G ruppe, als auch in der K G unauffällig. Sie entsprechen den N ormwerten, die Filipp und Freudenberg [1989] im SA M-Fragebogen für ihre G esamtstichprobe angeben. Inferenzstatistisch unterscheiden sich A sthmatiker weder in privater noch in öffentlicher Selbstaufmerksamkeit von der K G. Von den C O B-Patienten unterscheiden sie sich zwar durch höhere Werte, dies läuft aber hinsichtlich der privaten Selbstaufmerksamkeit der $\mathrm{H}$ ypothese entgegen. Für die öffentliche Selbstaufmerksam- 
keit ist angesichts der Normabweichung bei den COB-Patienten (s.u.) die dritte H ypothese ebenfalls zurückzuweisen.

Da man argumentieren könnte, daß die $H$ ypothese nur für «psychosomatische Patienten» relevant ist und bei Patienten mit «extrinsischem» und «gemischtförmigem» A sthma eine Beteiligung psychischer Faktoren am Krankheitsgeschehen weniger wahrscheinlich ist, haben wir zusätzlich untersucht, ob sich die Hypothese 3 spezifisch für die Subgruppe mit «intrinsischem» A sthma bestätigen läßt. D eren M ittelwerte in den Selbstaufmerksamkeitsskalen unterscheiden sich jedoch nicht signifikant von den beiden anderen A sthma-Subgruppen oder von der Kontrollgruppe.

Weiterhin ergibt sich für die CO B-Patienten ein unerwartetes Ergebnismuster. Sie weisen signifikant niedrigere Werte als die beiden anderen U ntersuchungsgruppen auf. Vergleicht man ihre Werte explorativ mit denjenigen der gesunden G esamtstichprobe von Filipp und Freudenberg [1989], so sind sie auch in diesem Fall signifikant geringer $(\mathrm{t}(230)=1,93$; $p<0,05$; einseitige Testung).

\section{Diskussion}

Zur Prüfung unserer ersten $\mathrm{H}$ ypothese, wonach A sthmatiker eine ungenauere Interozeption aktueller A temwegsobstruktionen aufweisen, haben wir A sthma- und CO B-Patienten untersucht. D ie A bweichung der subjektiven $E$ inschätzung von der tatsächlichen A temwegsfunktion war dabei in beiden $\mathrm{G}$ ruppen statistisch gleich groß. Die $\mathrm{H}$ ypothese ist somit in ihrer ursprünglichen Form zurückzuweisen.

$D$ ie $D$ atenexploration erbrachte allerdings einen interessanten und replikationswürdigen B efund: A sthmatiker weichen in R ichtung einer Ü berschätzung der O bstruktion, CO B -Patienten tendenziell in $\mathrm{R}$ ichtung einer $\mathrm{U}$ nterschätzung der $\mathrm{O} b$ struktion vom zu erwartenden U rteil ab. Wir werden unten ausführen, daß sich dies aus verhaltensmedizinischer Sicht gut interpretieren läßt. Zunächst ist aber kritisch zu unterstreichen, daß es sich bei diesem B efund lediglich um ein exploratives $D$ atum handelt. Ferner bedarf auch die Validität des von uns eingesetzten Verfahrens zur Interozeptionsgüte aufgrund der Motivationsabhängigkeit bei der Peak-Flow-Messung [M aß et al., 1989] noch weiterer B estätigung. Für zukünftige Studien wäre es deshalb wünschenswert, wenn ein zwar aufwendigeres, aber auch gegenüber dem Spirometer reliableres Verfahren wie die G esamtkörperplethysmographie angewendet werden könnte.

Weiterhin ist für die Zukunft die U ntersuchung einer atemwegsgesunden Kontrollgruppe zu fordern. E s könnte auf dieser B asis ermittelt werden, ob G esunde über eine genaue Interozeption ihrer A temwegsfunktion verfügen oder ob es nicht auch bei $\mathrm{G}$ esunden zu gewissen Fehlurteilen kommen kann. Im Vergleich mit den Werten Gesunder wären die E rgebnisse der beiden atemwegserkrankten Gruppen dann noch besser begründet - und nicht nur an einem theoreti- schen Wert orientiert - als «Ü berschätzung» oder «U nterschätzung» der O bstruktion zu interpretieren.

Unsere zweite $\mathrm{H}$ ypothese, nach der bei A sthmatikern die Selbstwahrnehmung von atemwegsbezogenen Symptomen eingeschränkt ist, findet sich teilweise bestätigt. In der Skala FWK S-A weisen diese Patienten geringere Werte auf als beide Vergleichsgruppen, wobei nur gegenüber der Kontrollgruppe der U nterschied signifikant wird. Der letztgenannte Befund entspricht inhaltlich der Erwartung einer Aufmerksamkeitsabwendung und ergänzt die zur $H$ ypothese 1 berichteten E rgebnisse: A sthmatiker sind nicht nur in der Interozeption von atemwegsbezogenen O bstruktionen ungenau, sie machen auch in einem Fragebogen zur Selbsteinschätzung der Symptomwahrnehmung entsprechende A ngaben. M an könnte diesen Befund dahingehend interpretieren, daß ihre Aufmerksamkeitsabwendung bis zu einem gewissen $\mathrm{G}$ rad bewußt repräsentiert ist.

$\mathrm{O}$ b die gefundenen $\mathrm{U}$ nterschiede spezifisch für die atemwegsbezogene Symptomselbstwahrnehmung sind, sollte der E insatz der Skala FWKS-KS - einer Skala für allgemeine körperliche Symptome - klären. H ierbei zeigt sich eine geringere Symptomwahrnehmung bei A sthmatikern im Vergleich zu den beiden anderen $\mathrm{G}$ ruppen. Wenn also mit «dem K örper etwas nicht stimmt», so nehmen A sthmatiker - ihrer Selbsteinschätzung nach - dies weniger wahr als CO B -Patienten und atemwegsgesunde $R$ ehabilitationspatienten. $D$ ies gilt allerdings nicht für Symptome des $\mathrm{G}$ astrointestinaltraktes.

$M$ ittels des Fragebogens zur dispositionellen Selbstaufmerksamkeit schließlich sollte die H ypothese (3) geprüft werden, daß A sthmatiker geringere private und höhere öffentliche Selbstaufmerksamkeit aufweisen, daß sie also ihre Wahrnehmung nicht nur von körperlichen Symptomen, sondern generell von internalen, auch psychischen A spekten, abwenden und gemäß Sachse und R udolph [1992] statt dessen eher auf M einungen und Werthaltungen anderer achten (hohe öffentliche Selbstaufmerksamkeit). Beide Teilhypothesen sind zurückzuweisen, da die von uns untersuchten A sthmatikern in beiden genannten Skalen des SA M -Fragebogens keine auffälligen Werte aufweisen. D as gilt auch für die Subgruppe der Patienten mit «intrinsischem» A sthma.

A uffällig niedrige Werte in privater und öffentlicher Selbstaufmerksamkeit, unterhalb des Normbereichs, zeigen hingegen die CO B -Patienten. Wenn man in B etracht zieht, daß sich ein $\mathrm{G}$ roßteil der COB-Patienten aus $\mathrm{R}$ auchern rekrutiert, ist der B efund im Z usammenhang mit der von H ull [1981, 1986] vorgebrachten $\mathrm{H}$ ypothese $\mathrm{zu}$ interpretieren, daß Suchtmittel zur Reduktion oder Vermeidung von Zuständen privater Selbstaufmerksamkeit eingesetzt werden.

A n dieser Stelle ist kurz auf weitere methodische B egrenzungen der vorliegenden Studie hinzuweisen. Einen kritischen Punkt sehen wir in der eingeschränkten R epräsentativität der von uns untersuchten Stichproben. Sie weisen ein unterdurchschnittliches Bildungsniveau und ein überdurchschnittliches Alter auf (s. o.). Ferner könnten die hinsichtlich der 
Symptomselbstwahrnehmung gefundenen U nterschiede stichprobenabhängig sein: Die Kontrollgruppe wurde ebenfalls aus $\mathrm{K}$ linik-Patienten rekrutiert, und diese könnten zum B eispiel körperliche Symptome verstärkt wahrnehmen. A us den genannten Gründen sind weitere replikative Studien wünschenswert.

A bschließend möchten wir unter verhaltensmedizinischer Perspektive eine integrierende Interpretation anbieten, die angesichts der noch geringen Datenbasis - als eher hypothesengenerierend aufzufassen ist.

Für die G ruppe der A sthmatiker ergibt sich dabei folgendes B ild: A sthmatiker wenden ihre A ufmerksamkeit von potentiell unangenehmen $K$ örperprozessen $a b$, insbesondere (aber nicht nur) im B ereich der A temwege. Wenn sie - wie im Tiffeneau-M anöver - sich einer B eschäftigung mit ihrer A tmung aber nicht entziehen können, so neigen sie aufgrund ihrer im A Iltag verminderten $\mathrm{K}$ örperselbstbeobachtung zu ungenauen und überschätzenden U rteilen über eine vorliegende A temwegsobstruktion.

Die Tendenz, unzureichend auf internale Informationen zu fokussieren, ist bei A sthmatikern offenbar spezifisch für K örperprozesse; eine generell verminderte private Selbstaufmerksamkeit findet sich nicht. D ie E rgebnisse widersprechen damit denjenigen Sachses [unter anderem Sachse und Rudolph, 1992] an einer gemischten psychosomatischen Stichprobe; sie liefern keine $\mathrm{H}$ inweise, daß A sthmatiker diesbezüglich A bweichungen aufweisen. Wie Sachse [1994] an anderer Stelle ausführt, gibt es eben kein «psychosomatisches Wahrnehmungsdefizit»; eine Wahrnehmungsabwehr bestehe nur bezüglich bedrohlicher Themen. U nsere E rgebnisse sprechen dafür, daß das, was A sthmatiker bedroht, der A sthmaanfall selbst ist.

I $\mathrm{m} \mathrm{H}$ inblick auf die B ehandlung haben wir es bei dem aufgezeigten Bild nach Auffassung verschiedener Autoren [z.B. Lehrer, 1998] mit dem (gegenüber einer U nterschätzung) weitaus günstigeren Fall zu tun, da eine Ü berschätzung von A temsymptomen eher der rechtzeitigen Bereitstellung gegenregulativer $M$ aßnahmen dient. $D$ ies wird insbesondere dann als günstig angesehen, wenn die Ü berschätzung sich auf die A temorgane beschränkt und nicht hinsichtlich anderer interozeptiver E reignisse generalisiert ist [L ehrer, 1998]. Wichtig scheint uns jedoch auch der Hinweis, daß nicht alle von uns untersuchten Patienten in der gleichen $\mathrm{R}$ ichtung von einer genauen E inschätzung abweichen. E ine bessere differentielle Ausrichtung von Programmen zur Steigerung der A tmungssensibilität auf U nter- oder Ü berschätzer könnte deshalb ein sinnvoller nächster Schritt sein. D ie mit U nterschätzung und Ü berschätzung verbundenen psychologischen Prozesse dürften ebenso unterschiedlich sein wie ihre Konsequenzen. N atürlich ist U nterschätzung angesichts dessen, daß die Mehrzahl der Todesfälle bei A sthma vermeidbar wären und häufig auf eine verzögerte Inanspruchnahme ärztlicher H ilfe zurückzuführen sind [Sly, 1989], ungleich gefährlicher; Ü berschätzung ist aber häufiger. Wie eingangs betont, kann allerdings auch letztere für das $\mathrm{K}$ rankheitsgeschen ungünstig sein, zumindest, wenn sie Ausdruck einer übersteigerten Furcht vor A nfällen ist oder zur Übermedikamentierung führt.

B etrachtet man die G ruppe der COB -Patienten, so gibt es (in der Selbsteinschätzung) keine Tendenz, die A ufmerksamkeit von körperbezogenen Signalen abzuwenden. Ü berprüft man diese Selbsteinschätzung in einem objektiven Interozeptionstest, so stellt man jedoch fest, daß COB-Patienten zumindest in der relativen Sekundenkapazität zu ungenauen $U$ rteilen im Sinne einer U nterschätzung ihrer aktuellen 0 bstruktion neigen. Zusammen mit dem B efund, daß sie seltener als die beiden anderen $\mathrm{G}$ ruppen ihre A ufmerksamkeit auf private oder öffentliche A spekte des Selbst lenken, kann dies als motivierte Tendenz aufgefaßt werden: $R$ auchen als häufigste $U$ rsache chronisch obstruktiver B ronchitis wird eingesetzt, um Selbstaufmerksamkeit zu verringern [Hull, 1981; 1986]; Selbstaufmerksamkeit ist aber nötig, um valide $A$ ngaben über Interozeption zu machen [z.B. Gibbons et al., 1979]. Der Selbsteinschätzung von COB-Patienten (im FWKS), sie würden Körpersignale deutlich (zumindest deutlicher als A sthmatiker) wahrnehmen, ist im Licht der übrigen B efunde somit zu mißtrauen: $\mathrm{H}$ andelte es sich um valide Aussagen, müßten entsprechende Wahrnehmungen geeignet sein, die gesundheitsschädliche Wirkung des Nikotinkonsums bewußt zu machen. Verhaltensexperimente und Interozeptionsübungen könnten eingesetzt werden, um COB-Patienten mit hier möglichen Verleugnungs- und Vermeidungstendenzen zu konfrontieren.

E ine alternative Interpretation kommt allerdings ohne die A nnahme motivationaler Prozesse aus: Im Unterschied zu A sthma handelt es sich bei COB nämlich um das Vorliegen dauerhafter Obstruktionen. Dies könnte eine Verschiebung des E rwartungswertes (für Obstruktionen bzw. für freien A temfluß) zur Folge haben; das G efühl für eine unbeeinträchtigte A temwegsfunktion ist verlorengegangen, und die andauernd gegebene Sollwertabweichung wird für das Normale gehalten. Auch dies könnte die «U nterschätzung» der O bstruktionen erklären. Interozeptionstraining und andere $\mathrm{M}$ aßnahmen der sekundären und tertiären Prävention, die dem Aufbau einer besseren Gesundheitswahrnehmung dienen, wären jedoch auch in diesem Fall indiziert.

\section{Dank}

Wir danken M itarbeitern und Patienten der Rehabilitationsklinik «A $\mathrm{m}$ Taunus» in B ad Soden herzlich für die gute Kooperation. 


\section{Literatur}

Dahme B, König R, Nußbaum B, R ichter R: Haben A sthmatiker Defizite in der Symptomwahrnehmung? Quasi-experimentelle und experimentelle Befunde zur Interozeption der A temwegsobstruktion. Psychother Psychosom M ed Psychol 1991; 41:490-499.

E rdmann G, Janke W: Fragebogen zur Wahrnehmung körperlicher Symptome. Psychologisches Institut der U niversität D üsseldorf, 1975.

Fenigstein A, Scheier M F, B uss A H : Public and private self-consciousness: A ssessment and theory. J Consult Clin Psychol 1975;43:522-527.

Ferlinz R (H rsg): Internationaler Konsensus-B ericht zur D iagnose und B ehandlung des A sthma bronchiale. Pneumologie 1992;47:247-288.

Filipp S-H, Freudenberg E : D er Fragebogen zur E rfassung dispositioneller Selbstaufmerksamkeit (SA M -Fragebogen). G öttingen, H ogrefe, 1989.

Filipp S-H, K lauer T, Ferring D: Self-focused attention in the face of adversity and threat; in $\mathrm{K}$ rohne $\mathrm{H}-\mathrm{W}$ (ed): A ttention and A voidance. Seattle, $\mathrm{H}$ ogrefe und H uber, 1993, pp 267-294.

Gerhards F: Emotionsausdruck und emotionales Erleben bei psychosomatisch K ranken. O pladen, Westdeutscher Verlag, 1988.

G ibbons FX, Carver CS, Scheier M F, H ormuth SE : Self-focused attention and the placebo effect: Fooling some people some of the time. J Exp Soc Psychol 1979;15: 263-274.

H euser J : Der Zusammenhang von Selbstaufmerksamkeit, Schmerzwahrnehmung und Interozeption bei A Ikoholikern und G esunden. U nveröff. D iplomarb., U niversität B onn, 1991.

H ull J G : A self-awareness model of the causes and effects of alcohol consumption. J A bn Psychol 1981;90: 586-600.

H ull J G, Y oung R D, J ouriles E : A pplications of the self-awareness model of alcohol consumption: Predicting patterns of use and abuse. J Pers Soc Psychol 1986; 51:790-796.

Könning J, Schon M, Gebert N, Schmidt S, Szczepanski R: Körperselbstwahrnehmung bei $A$ sthma als Voraussetzung für Selbstregulation. Verhaltensther psychosoz Praxis 1996;28:217-230.

Kosarz P: E in verhaltensmedizinisches M odell des A sthma bronchiale; in Zielke M (H rsg): $\mathrm{H}$ andbuch stationäre Verhaltenstherapie. Weinheim, Psychologie-VerlagsU nion, 1994, pp 640-648.

Lehrer P: E motionally triggered asthma: A review of research literature and some hypotheses for self-regulation therapies. A ppl Psychophysiol Biofeedback 1998;23: 13-41.

Löllgen $H$, Dirschedl $P$ : Chronische Lungenerkrankungen und Sport. Internist 1992;33:142-149.

M aß R, Richter R, D ahme B: Zur Biofeedback-Therapie des A sthma bronchiale. Prax klin Verhaltensmed R eha 1989;6:68-73.

M erz J : SA F : Fragebogen zur M essung von dispositioneller Selbstaufmerksamkeit. D iagnostica 1986;32: 142-152.

Q uanjer PH: Standardized lung function testing. European Community for Coal and Steel, Luxembourg, 1983.

R eusch A : A sthma bronchiale versus chronisch obstruktive Bronchitis: Interozeptionsgüte, Aufmerksamkeitsorientierung und Emotionsausdruck. Unveröffentl. D iplomarbeit, J ohann-Wolfgang-G oethe-U niversität, Frankfurt am M ain, 1997.

Sachse R : H erzschlagwahrnehmung bei psychosomatischen Patienten: A bwendung der A ufmerksamkeit von eigenen Körperprozessen. Psychother Psychosom M ed Psychol 1994:44:284-292.

Sachse R: Der psychosomatische Patient in der Praxis. Stuttgart, Kohlhammer, 1995.

Sachse R, Rudolph R : Selbstaufmerksamkeit bei psychosomatischen Patienten. Z klin Psychol Psychopath Psychother 1992;40:148-164.

Sly R M : M ortality from asthma. J A llergy Clin Immunol 1989;82:421-434.

Stout $\mathrm{C}$, Kotses $\mathrm{H}, \mathrm{Creer} \mathrm{JL}$ : Improving recognition of respiratory sensations in heathy adults. Biofeedback Selfregulation 1993;18:79-92.

Stout C, Kotses H, C reer J L : I mproving perception of air flow obstruction in asthma patients. Psychosom M ed 1997;59:201-206. 